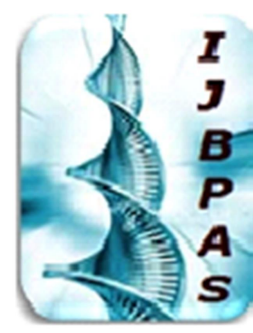

International Journal of Biology, Pharmacy and Allied Seiences (IJBPAS) 'A Bridge Betusen Caboratory and QRader'

WwW.iibpas.com

\title{
REVIEW ON FAVIPIRAVIR
}

\section{KEERTHI KATTEPOGU*, GOLLAPALLI NAGARAJU, PRACHET PINNAMANENI AND NADENDLA RAMA RAO}

Department of Pharmaceutical Analysis, Chalapathi Institute of Pharmaceutical sciences, Lam, Guntur 522034

*Corresponding Author: Keerthi Kattepogu: E Mail: kattepogu.keerthi7@gmail.com; Mob.: 9949630973

Received $18^{\text {th }}$ Feb. 2021; Revised $17^{\text {th }}$ Mar. 2021; Accepted $15^{\text {th }}$ April. 2021; Available online $1^{\text {st }}$ Dec. 2021 https://doi.org/10.31032/IJBPAS/2021/10.12.5738

\begin{abstract}
The present work converse the outline of Favipiravir for its effective role against the virus. The central drugs standard control organization granted accelerated approval to Glenmark pharmaceuticals to market Favipiravir in mild-to-moderate COVID-19 on June 19, 2020, for restricted use. It is the right time to take policy decision and frame guidelines on how to handle emergency approvals for medicinal products in grave situations such as the COVID19 pandemic in India. This paper gives the information about the complete drug profile of Favipiravir like structure, mechanism of action, kinetics, drug interactions, clinical trials data, analysis of drug and adverse effects. It has good antiviral activity, because of this it is extensively studied for its probable use in treatment for this corona virus. Favipiravir was first introduced in Japan in 2014 as a cure for influenza after the research on this medicine also revealed that it is a broad spectrum medicine and can be used in treatment of many viral diseases.
\end{abstract}

\section{Keywords: Favipiravir, COVID-19, SARS-CoV-2}

\section{INTRODUCTION}

COVID-19 pandemic due to severe acute respiratory syndrome corona virus 2 (SARS-coV-2) infection has already resulted in more than 6 lakhs death worldwide [1]. The common symptoms [2] of COVID-19 are as follows-

- Fever

- Dry cough 
- Fatigue

- Body pains

- Nasal congestion

- Conjunctivitis

- Sore Throat

- Loss of taste and smell

- Head Ache

Favipiravir was developed by the Fujifilm Toyama Chemical Company and is licenced in Japan and china. The anti-viral drug Favipiravir is a derivative of pyrazine carboxamide and its chemical formula is 6fluoro-3-hydroxy- 2-pyrazinecarboxamide [3]. Its brand name is Avigan [22, 23]. The active compound of this drug is favipiravir ibofuranosyl-5'triphosphate (T-705-RTP) which is produced by intracellular ribosylation and phosphorylation. It selectively inhibits RNA dependent RNA polymerase (RdRp), an enzyme needed for RNA viral replication within human cells. It functions as a purine analogue and is incorporated instead of guanine and adenine. It has broad spectrum of activity towards RNA viruses (Influenza, Rhino, and Respiratory syncytial virus etc) but not against DNA viruses (such as Herpes) [3]. The human body is susceptible to infection from a number of microbes including bacteria, fungi and viruses etc. Among all these the virus is the trickiest microbe which harms the human body. Due to the nature of the viruses being living and replicating in the human body and outside the body they remain in crystallized form. This makes it very difficult to develop a cure or vaccine for maximum viral disease like AIDS, COVID-19 etc. Middle East Respiratory Syndrome (MERS) and severe Acute Respiratory Syndrome (SARS) also come under the umbellia of coronavirus. COVID-19 is a recently discovered disease that is claimed to have recorded its first case in December 2019 in the city of Wuhan, China [2].

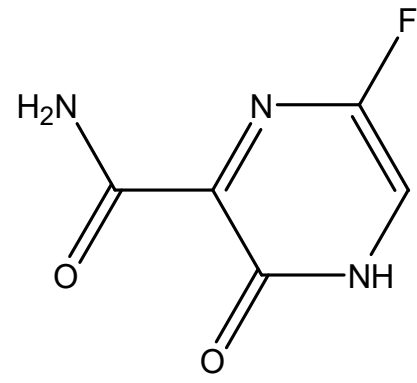

Figure 1: Structure of Favipiravir

\section{Mechanism of Action:}

Favipiravir is a pyrazinecarboxamide derivate prodrug, which isribosylated and phosphorylated inside the cell and forms favipiravir ibofuranosyl-5' -triphosphate, which is an active metabolite. The system of activity of favipiravir is novel contrasted with existing flu antivirals that principally forestall section and exist of the infection from cells. The dynamic favipiravir-RTP specifically restrains RNA polymerase and forestalls replication of the viral genome. There are a few theories regarding how favipiravir-RTP communicates with RNA subordinate RNA polymerase (RdRp). 
Some investigations have demonstrated that when favipiravir-RTP is joined into a beginning RNA strand, it forestalls RNA strand stretching and viral proliferation. It has a unique mechanism of action. It is converted into an active phosphoribosylated form (favipiravir-RTP) in cells and recognized as a substrate by viral RNA polymerase, thereby inhibiting RNA polymerase activity.

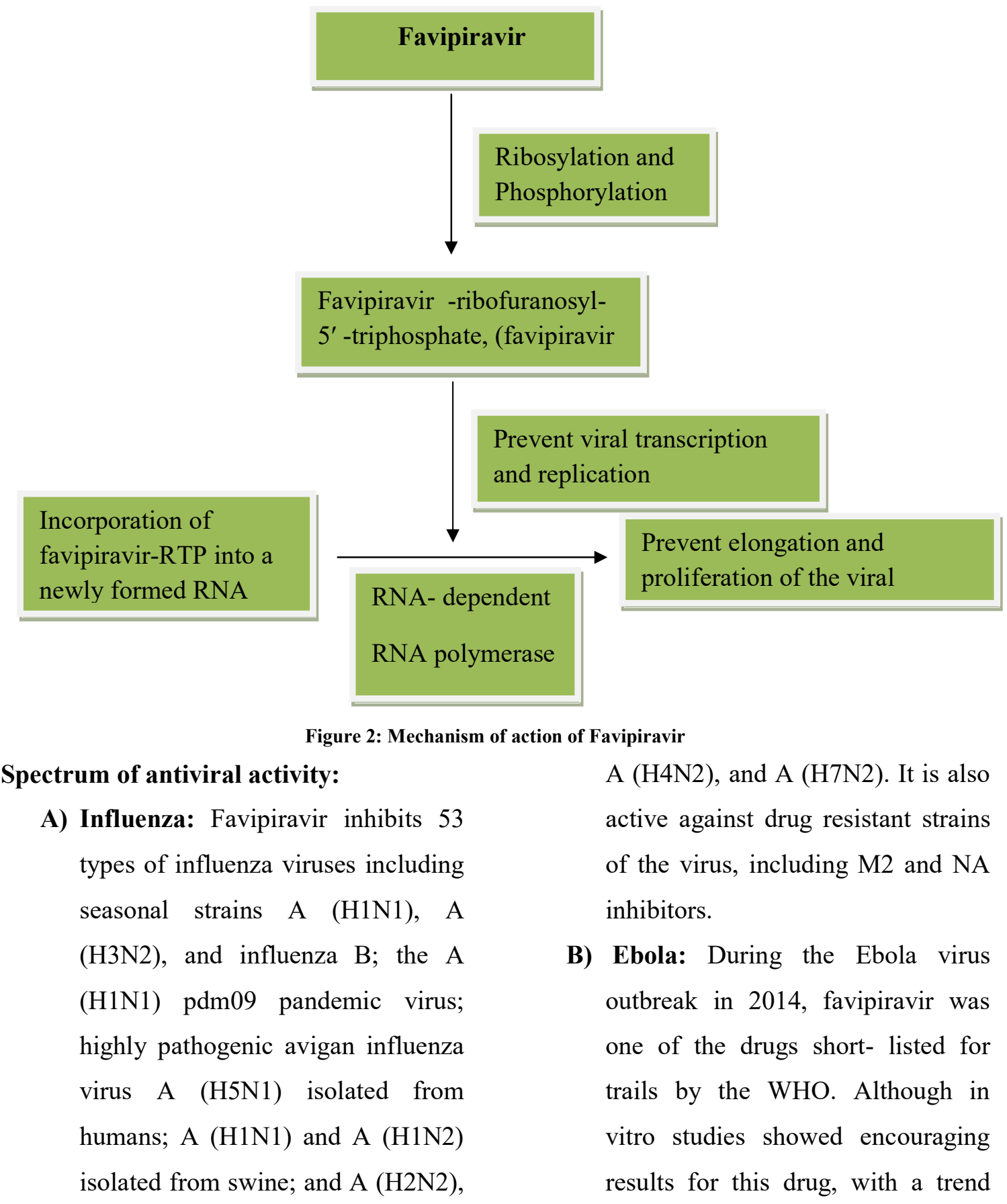


toward survival benefit showed by clinical studies, conclusive evidence of benefit was never found. In the JIKI multicenter trail conducted in 126 patients with Ebola, Favipiravir in an initial loading dose of $6000 \mathrm{mg}$ followed by $2400 \mathrm{mg}$ /day for 9 days was shown to have some effect in patients with medium to high viremia but not in those with more severe viremia $(\mathrm{Ct}$ value $<20)$.

\section{C) Activity against other pathogenic} RNA viruses: In addition to its activity against influenza and Ebola viruses, Favipiravir has been found to have therapeutic efficacy in cell culture and mouse models of Arena virus, bunya virus, filovirus, West Nile virus, yellow fever virus, footand-mouth-disease virus, and Lassa virus including agents causing viral hemorrhagic fevers and encephalitis.

Pharmacokinetics and pharmacodynamics:

Favipiravir is administered as a prodrug. It has an excellent bioavailability (94\%), 54\% protein binding, and a low volume of distribution (10-20 L). it reaches Cmax within $2 \mathrm{~h}$ after a single dose. Both Tmax and half-life-increase after multiple doses. Favipiravir has a short half-life (2.0-5 h) leading to rapid renal elimination in the hydroxylated form. Elimination is mediated by aldehyde oxidase and marginally by xanthine oxidase. Favipiravir exhibits both, dose dependent and time dependent pharmacokinetics. It is not metabolized by the cytochrome p450 system, but inhibits one of its components (CYP2C8). Thus, it needs to be used with caution when coadministered with drugs metabolized by the CYP2C8 system.

Absorption: The bioavailability is about $94 \%$ and the Cmax is $51.5 \mu \mathrm{g} / \mathrm{ml}$. Favipiravir has many interactions with 200 types of food items that decreases Cmax so to compensate the dose needs to be increased. Drug-drug interactions can lead to serious conditions, especially in patients with co morbidities and complications such as hypertension, diabetes, and cardio vascular disease.

Distribution: As Favipiravir is a prodrug and its activation occurs in the liver, also the volume of distribution will be different between prodrug (Favipiravir) and active metabolite (Favipiravir-RTP) there are still ongoing experiments at the clinical trial stage. However, for Favipiravir, the volume of distribution is $15-20 \mathrm{ml}$.

Metabolism: Normally, all the antivirals undergo hydroxylation primarily by aldehyde oxidase and also by xanthine. From studies, it is found that human liver microsomes are sites for hydroxylation that forms Favipiravir hydroxylate at a range of aldehyde oxidase activity by 12 times to maximum. Along with hydroxylating, 
glucuronate conjugate is also observed in human plasma.

Excretion: It is excreted through urine in hydroxylate from also a little amount of unchanged drug in the ratio of $53.1 \%: 0.8 \%$, respectively.

\section{Clinical trials in COVID-19:}

Over the past few months, clinical studies have been performed all over the world to assess the efficacy of Favipiravir in the management of COVID-19. The major clinical trials studies are summarized here China:

Chen.et.al. had conducted a prospective, open-label multicentric trial in china to compare two treatment arms in the management of clinically confirmed COVID-19 (maximum duration of symptom onset before randomization 12 days).Conventional therapy plus umifenovir (Arbidol) (200mg thrice a day) for 7 days (extendable to 10 days). The study comprised 240 patients with 1:1 randomization to both groups. The authors found that the clinical recovery rate at day 7 did not differ significantly between the two groups (61.21\% for umifenovir, 95\% CI: -0.0305 to $0.2213, \mathrm{p}=0.1396)$. There was no significant differences between the two groups in terms of the incidence of auxiliary oxygen therapy or noninvasive mechanical ventilation. The two groups were comparable in terms of all cause mortality, dyspnea after taking medications, and respiratory failure. All these were considered mild side effects.

Japan:

A Japanese observational study group recorded the details of hospitalized COVID-19 patients in Japan to assess the safety and efficacy of Favipiravir. In more than $90 \%$ of cases, Favipiravir was administered at a dose of $1800 \mathrm{mg}$ orally on day 1 followed by $800 \mathrm{mg}$ twice daily on subsequent days. The median duration of therapy was 11 days. Rates of clinical improvement at 7 and 14 days were $73.8 \%$ and $87.8 \%, 66.6 \%$ and $84.5 \%$ and $40.1 \%$, $60.3 \%$ for mild, moderate, and severe disease, respectively. Favipiravir in combination with Nafamostat was found to be useful in a small case series consisting of 11 serious patients with COVID-19 in Japan. The median age, time from symptom onset to admission in the ICU, and $\mathrm{paO}_{2} / \mathrm{FiO}_{2}$ ratio on admission were 68 years(IQR60-69), 8 days (IQR 7-11), and 131 (IQR114-198) respectively. All patients needed oxygen therapy, eight patients $(73 \%)$ needed invasive mechanical ventilation, and 3 patients $(27 \%)$ needed extracorporeal membrane oxygenation (ECMO). Of the 11 patients, 7 were successfully weaned from mechanical ventilation, was still in the hospital at the time the paper was published. 


\section{Indian trial:}

A randomized, multicenter, open-labeled clinical trial in Indian patients has just been completed, with results expected to be published soon. This trial evaluated the efficacy and safety of Favipiravir in patients hospitalized with mild to moderate COVID-19 infection. Conducted in hospitals across India, 150 patients were randomized, with 72 to the Favipiravir arm and 75 to the SOC arm. Those in the Favipiravir arm received $3600 \mathrm{mg}$ on day 1, then $1600 \mathrm{mg}$ on days 2-14. Daily nasopharyngeal swabs were collected from all participants till two consecutive swabs were negative. The primary end points analyzed in this study were clinical cure rates as determined by the treating physician with recovery of fever, respiratory rate, oxygen saturation, and cough relief. The trial also looked at other secondary end points such as time from randomization to initial requirement of high flow supplemental oxygen or ventilator support and time from randomization to hospital discharge.

\section{The USA:}

The research team at Stanford medicine have recently commenced a double-blind, placebo-controlled trial (Favipiravir $\mathrm{v}_{\mathrm{s}}$. placebo for 10 days) to assess the utility of Favipiravir in reducing symptoms and the duration of viral shedding in outpatients with COVID-19. About 120 patients are expected to be enrolled beginning July 6 , 2020.

\section{Influence of strains in vaccine:}

When different strains are evolving, it becomes difficult to invent vaccines as the vaccine provides long-term protection. Normally dose, DNA, and MVA-vaccines encode $S$ glycoprotein neutralizes antibodies also $\mathrm{N}$ protein (induce longlived memory T cells) in the case of MERS and SARS. So now, this aspect become a potentially viable alternative long term $\mathrm{T}$ cell immunity against in coronavirus.

The strain that is inert will preserve the features that will provide immunity against a whole group of variants which make it easier to study and make a perfect unique target in discovering vaccines against it. As there are more mutations in SARS-CoV-2, an RNA vaccine can be more powerful than involves supplementing a similar section of the genome in the form of RNA into the host body.

It became a challenge for scientists all over the world to discover vaccines with no side effects after performing many experiments in different phases and by a comparative study between many drugs they have discovered Favipiravir is the most effective drug in treating SARS-CoV-2 such as dexamethasone, lopinavir/ritonavir, Ivermectin, and remdesivir.

Phases in development of vaccine: when they find a new vaccine towards any 
disease, they need to undergo the following process before getting that vaccine into the market:

* Pre-clinical tests: this includes the primary stage first done in vitro (test tube or cell culture) and also in vivo (animals). This process helps testing preliminary efficacy, toxicity, pharmacokinetics, and safety information of vaccines. When this stage passes, then they can try for the next step on humans.

* Phase-1: In this phase, they conduct trails in 20-80 humans for several months watching regularly. This phase has significance in tracing out the highest dos, side effects, and the best route of administration.

* Phase-2: Here, experiments will be done on 25-100 people that they categorize them into different groups and compare the studies between them and intervein the results.

* Phase-3: In this site, the study size is increased to 3000 participants and this is the last phase of clinical trials and when this passes that they give the approval to release the drug into the market.

* Approval: After phase-3 trials, the candidate who performs the experiments applies for approval of his vaccine to release it into the market. However, when there occurs a pandemic situation that vaccines can be approved under emergency authorization.

\section{Favipiravir in SARS-CoV-2:}

The Drug Controller General of India has approved Favipiravir as emergency medical use on June 19, in India. Favipiravir is the first oral approved medicine in India for treating SARS-CoV-2 launched by Glenmark Pharmaceuticals with the name FabiFlu for conducting phase 3 clinical trials in patients with mild-to-moderate symptoms.

\section{Side effects/Adverse effects:}

The Japanese study discussed previously found that adverse reactions were seen in around $20 \%$ of the patients who received Favipiravir (at a dose lower than approved for COVID-19). The adverse effects were relatively minor and included hyperuricemia and diarrhea in $5 \%$ of the participants and reduced neutrophil count and transaminitis in $2 \%$ of the participants. One study showed occurrence of psychiatric symptoms in association with Favipiravir. Effect of Favipiravir in QTc prolongation is still uncertain, with some pharmacodynamic studies suggesting a appositive association, but a Japanese study suggesting otherwise. Overall, Favipiravir has a good safety profile, as was confirmed by a large systematic review. In the following sections, we give a brief overview of the adverse effect profile of this drug. 


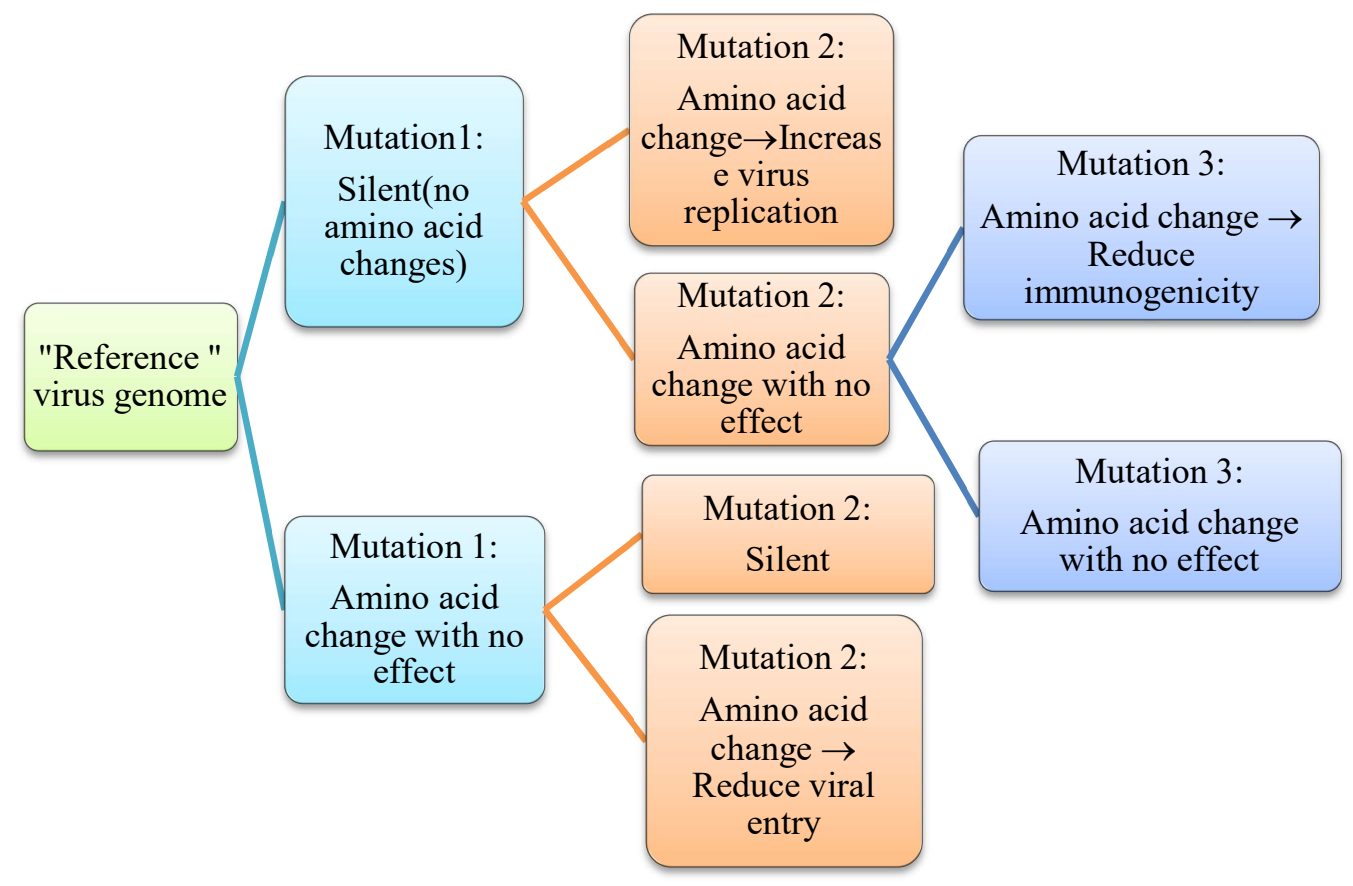

\section{Hyperuricemia:}

Favipiravir use results in a dose-dependent increasing trend in the prevalence of Hyperuricemia. A systematic review conducted by Pilkington et al. found similar trends across multiple studies. This is however not associated with clinical manifestations. There has been no evidence that Hyperuricemia caused by Favipiravir leads to clinical manifestations; however, longer follow-up periods would be required to fully assess this risk.

\section{Teratogenicity:}

There is evidence that Favipiravir has a teratogenic potential and embryotoxicity. The Japanese drug safety bureau approval advises that Favipiravir be given a strong warning against use in women of reproductive age and recommends precautionary statements on packaging and prescription alerts. The bureau also recommends that Favipiravir should be avoided where alternative drugs could be used. Effective contraceptive methods during and for 7 days after the end of treatment need to be instructed to men who have received that treatment. Before Favipiravir is prescribed to women of child-bearing age. It is imperative to rule out pregnancy with a negative urine pregnancy test.

\section{Drug interactions:}

Pyrazinamide: Concombitant use of Pyrazinamide with Favipiravir increases the levels of uric acid. Regular uric acid level 
monitoring is mandatory when these drugs are used together.

Repaglinide: Favipiravir inhibits the metabolism of Repaglinide through the CYP2C8 pathway, thus increasing its potential to cause toxicity (hypoglycemia, headache, increase incidence of upper respiratory tract infections, etc).cautious concomitant use is recommended.

Theophylline: Theophylline increases the blood levels of Favipiravir and adverse reactions to Favipiravir may occur.

Famciclovir, sulindac: Efficacy of these drugs may be reduced when coadministered with Favipiravir.

Acyclovir: Acyclovir may delay the conversation of Favipiravir into the active moiety, thus reducing its antiviral efficacy.

\section{Dose Recommendation:}

The estimation of half-life of Favipiravir is around 2-5.5h. It undergoes hydroxylation primarily by aldehyde oxidase and by xanthine oxidase. Favipiravir can start with an initial dose of $1600 \mathrm{mg}$ twice a day, followed by $600 \mathrm{mg}$ twice for a period of 7 10 days.

\section{Dose Adjustment of Favipiravir in renal} and hepatic dysfunction:

In people with eGFR $30-50 \mathrm{~mL} / \mathrm{min}$, the plasma level of Favipiravir was found to be about three times higher. Still, it is safe, but not preferred in people with eGFR $>20$ $\mathrm{mL} / \mathrm{min}$. Usually, no dose adjustment is required in renal impairment.

\section{Combination therapy:}

With methylprednisolone: when favipiravir, along with methylprednisolone, was administered in patients with a severe condition of COVID-19 and they are on the ventilator as they have SPO $2<93 \%$ which have been recorded well and even they do not require ventilator further but the administration should be started in early stage only to achieve favorable outcomes.

With umifenovir: Glenmark has approved that an antiviral combination can effectively be used in high viral loads in the early stage of the disease such as umifenovir that has that the combined effect has a comprehensive antiviral cover on the pre-entry and post-entry life cycle of SARS-CoV-2.

\section{Analytical methods:}

\section{HPLC:}

A rapid, simple, precise, accurate, and isocratic high performance liquid chromatography (HPLC) method has been developed for routine quality control of Favipiravir in pharmaceutical formulations. Separation was carried out by $\mathrm{C} 18$ column. The mobile phase was a mixture of $50 \mathrm{mM}$ potassium dihydrogen phosphate $(\mathrm{pH} 2.3)$ and acetonitrile $(90: 10, \mathrm{~V} / \mathrm{V})$ at a flow rate of $1 \mathrm{~mL} \mathrm{~min}^{-1}$. The ultra- violet (UV) detection and column temperature were $323 \mathrm{~nm}, 30^{\circ} \mathrm{C}$. The run time was $15 \mathrm{~min}$ under these chromatographic conditions. Linearity between peak area and 
Favipiravir concentration in the range of $10-100 \mu \mathrm{g} / \mathrm{ml}^{-1}$

\section{HPLC column screening for favipiravir on six different 100x 4.6mm columns:}

Initial column screening was carried out using gradient conditions $(5 \% \mathrm{~B}$ for 0.5 minutes, to $95 \% \mathrm{~B}$ over 10 minutes); however, due to lack of significant differences in retention, we elected to run under isocratic conditions to highlight the differences in retention across the six different stationary phases.

\section{LC-MS/MS:}

A method for the analysis of Favipiravir, remdesivir, chloroquine, hydroxychloroquine, lopinavir, ritonavir, and dexamethasone in plasma developed using $50 \mu \mathrm{l}$ of sample. The sample was precipitated with an internal standard containing solution, and supernatant was diluted prior to injection on an ACQUITY UPLC 1-Class/Xevo TQ-S micro IVD system. Separations were performed with the CORTECS T3, $2.1 \mathrm{~mm} \times 50 \mathrm{~mm}, 2.7$ $\mu \mathrm{m}$ column using gradient elution and a mobile phase comprised of ammonium formate, formic acid, and methanol.

\section{CONCLUSION}

Favipiravir is Anti-viral agent used for patients with COVID-19. The paper includes the complete description of the novel anti-viral medicine called Favipiravir which was first launched in 2014 in Japan and it was used to treat influenza which was caused by an RNA virus. This medicine being a potent drug for eradication of the RNA viruses has been potentially studied to treat other such viral infections like Ebola virus infection, bunya virus infection and even the corona virus infection that has become a pandemic recently. Also tells about the analytical work done so far on the particular drug, so far only one method was developed which gives the information about a lot of work need to be done. Therefore, we conclude that Favipiravir is the best medication for viral infections and being effectively used presently in COVID-19.

\section{Acknowledgment}

We are thankful to the management of Chalapathi institute of pharmaceutical sciences, Lam, Guntur, for providing necessary support, guidance and facilities.

\section{REFERENCES}

[1] Suman omana soman, A.V. Raveeendran., (2020). "Remdesivir and Favipiravir for COVID-19: An update," Annals of clinical cardiology. 2(2): page. 51-54.

[2] Dr. Shashank Tiwari \& Ms. Shreya Talreja., (2020). "Drug Review: Favipiravir," $\quad$ Science and Engineering Journal. 24 (9): page. 26-32.

[3] Suranjith L Seneviratne, Visula Abeysuriya, Sanjay de Mel, Ishan De Zoysa, Roshan Niloofa., (2020). 
"Favipiravir in Covid- 19," International Journal of Progressive Sciences and Technologies. 19(2): page. 143-145.

[4] C, Huang J, Cheng Z, Wu J, Chen S, Zhang Y et al., (2020). "Favipiravir versus Arbidol for COVID-19," A randomized Clinical Trial. MedRxiv.

[5] Furata Y, Komeno T, Nakamura T., (2017). "Favipiravir (T-705), a broad spectrum inhibitor of viral RNA polymerase," Proc Jpn Acad Ser B Phys Biol Sci. 93(7): page. 449-463.

[6] Yousuke Furuta, Brian B, Gowen, Kazumi Takahashi, Kimiyasu Shiraki, Donald F. Smee, Dale 1, Barnard., (2013). "Favipiravir (T705), a novel viral RNA polymerase inhibitor," Antiviral Research. 100(2): page.446-54.

[7] Arunkumar Radhakrishnan, Ruckmani arunachalam, Abinaya Elango., (2020). “Critiacal Review and analysis of approval of Favipiravir for restricted emergency use in mild- to- moderate COVID19," Journal of pharmacology \& pharmacotherapeutics. $11(1)$ : Page.1-7.

[8] Umang Agarwal, Reyma Raju, Zarir F. Udwadia., (2020). "Favipiravir: A new and emerging antiviral option in COVID-19," Medical Journal Armed Forces India. 76(4): page. $370-376$.

[9] Shaik Ali Hassan, Sumit Bhateja, Geetika Arora, Francis Prathyusha., (2020). "Can Favipiravir help in coronavireus treatment?," IP International Journal of Comprehensive and Advanced Pharmacology. 5(2): page. 73-76.

[10] Jin Z, Smith LK, Rajwanshi VK, Kim B, Deval J.,(2013). "The ambiguous base-pairing and high substrate efficacy of T- 705 (Favipiravir) ribofuranosyl 5' triphosphate towards influenza A virus polymerase," Plos One. 8(7)

[11] James Ives M. preliminary report of Favipiravir observational study in Japan released. NewsMedical.net., (2020). https://www.newsmedical.net/news/20200602/preli minary-report-of-FavipiravirObservational-Study-in-Japanreleased.aspx.

[12] Investigational antiviral study finds faster clinical Improvement in patients with COVID-19 Pharmacy Times., (2020).

[13] Pharmaceuticals and Medical Devices Agency., (2014). "Report on the Deliberation Results Avigan Japan," Evalution and 
Licensing Division, pharmaceutical and Food Safety Bureau.

[14] Kumangai Y, Murakawa Y, Hasunuma T., (2015). "Lack of effect of Favipiravir, a novel antiviral agent, on the QT interval in healthy Japanese adults," International .Journal of Clinical Pharmacology and Therapeutics. 53(10).

[15] Pilkington V, Pepperrell T, hill A., (2020). "A review of the safety of Favipiravir - a potential treatment in the COVID-19 pandemic?,". Journal of Virus Erad. 6(2): page. 45-51.

[16] Catherine M, Neil S, Brian G, Wayne N., (2020). "Favipiravir and Remdesivir Appear to be Realtively Unencumberred with Drug-Drug Interactions," Anesthesia Patient Safety Foundation.

[17] RDIF and ChemRar launch final stage of Favipiravir clinical trial (2020).

https://www.prnewswire.com/ae/n ews-releases/rdif-and-chemrarlaunch-final-stage-of-favipiravirclinical-trial-301064181.hyml.

[18] Ibrahim bulduk., (2020). "HPLCUV method for quantification of Favipiravir in pharmaceutical formulations," Acta

Chromatographica. 32(4):
[19] Ashley C. Banyard, Karen L. Mansfield, Guanghui Wu, David Selden, Leigh Thorne, Colin Birch et al., (2019). "Re-evaluating the effect of Favipiravir treatment on rabies virus infection," Vaccine. 37(33): page. 4686-4693.

[20] Vincent Mandelain, Jeremie Guedj et al., Favipiravir Pharmacokinetics in Nonhuman primates and insights for future efficacy studies of hemorrhagic fever viruses. Antimicrobial Agents and Chemotherapy; 2017 61(1).

[21] Hayden FG, Shindo N., (2019). "Influenza virus polymerase inhibitors in clinical development," Curr Opin Infect Dis. 32(2): page. 176-186.

[22] Pharmaceutical and medical devices agency: Avigan (Favipiravir) review report., (2016).

[23] Glenmark to Study Potential COVID-19 Drug Combination on Favipiravir, Umifenovir in India., (2020).

https://www.oneindia.com/india/glen mark-to-study-potential-covid-19drug-combinationon-favipiravirumifenovir-in-india-3094526.html. 